\title{
ARTERIAL PRESSURE IN NORMAL SERBIAN CHILDREN
}

\author{
BY \\ Dr. VLADISLAV BOGDANOVITCH, \\ Physician to the Anglo-Serbian Children's Hospital, Belgrade.
}

The question of arterial pressure in the normal child has been the subject of many studies, but there is no great uniformity in the results recorded. The facts that the various writers did not measure the arterial pressure in children of exactly the same age, nor use the same apparatus, explain the divergence in the results recorded. Further, hygienic conditions, feeding and climate in the various countries, play an important part in development, and thus exert an influence on the arterial tension in children even of the same age. Differences in technique, anatomical anomalies, and sometimes an insufficient number of examinations, account also for the varying results obtained.

In the present investigations, from 1925 until the end of 1932, there were examined 840 normal and healthy children of both sexes between the ages of 4 and 15 years; 230 of these children were patients in the AngloJugoslav Children's Hospital in Belgrade, 360 were school children of both sexes from 6 to 10 years, and 250 were school children from 10 to 15 years.

The children were all examined in the recumbent posture and the observations were made on the right arm. Two types of apparatus were used:- - the Vaquez-Laubry manometer (auscultatory method), and Pachon's oscillometer. With the Vaquez-Laubry apparatus armlets of two sizes were employed :- for children of 4 to 11 years one of $5 \mathrm{~cm}$., and for children of 11 to 15 years one of $12 \mathrm{~cm}$. The ordinary model of the Pachon oscillometer was employed with an armlet measuring $8 \mathrm{~cm}$. in width.

Vaquez - Laubry apparatus.-With this apparatus the maximum blood pressure in boys measured 101.5 to $122.5 \mathrm{~mm}$., and the minimum from 70 to $95 \mathrm{~mm}$. In girls the maximum pressure varied from 100 to $125 \mathrm{~mm}$., and the minimum from 72.5 to $85 \mathrm{~mm}$. The differential pressure measured about $30 \mathrm{~mm}$. (Table 1 and Chart I).

From a study of these results the following facts emerge. The maximum and minimum pressures in Serbian children of both sexes are inclined to increase as the child grows older. There is also a slight increase in the differential pressure, especially in girls after the age of 7 years. It is interesting to note, too, that there is a somewhat more definite increase in the maximum and minimum pressures between 12 and 14 years in both sexes, the age of puberty; and that the maximum pressure in girls of 13,14 
TABLE 1 .

Arterial tension, according to age, nieasured by the Vaquez-Laubry apparatus.

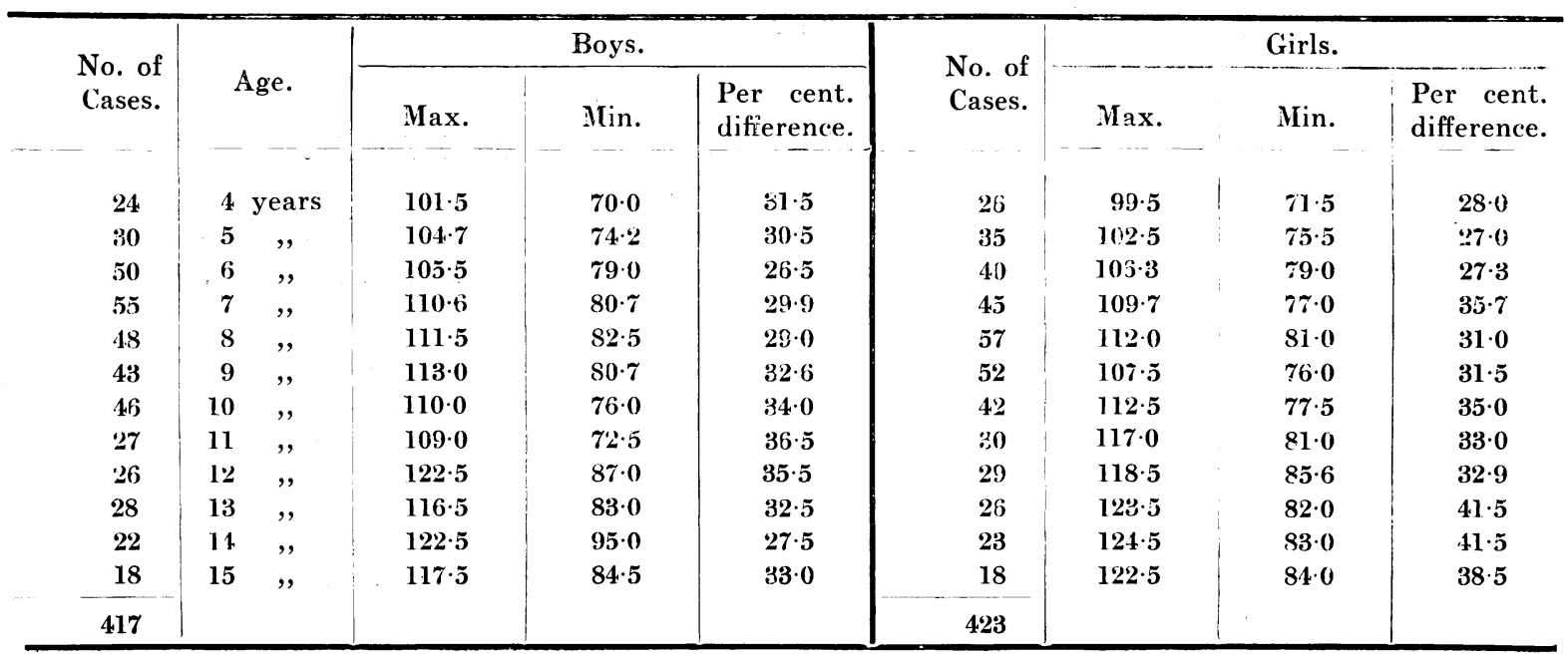

Medium value.

CHART I.

Boys.

Girls.

Pressure

$\mathrm{Mm} . \mathrm{Hg}_{\mathrm{g}}$.
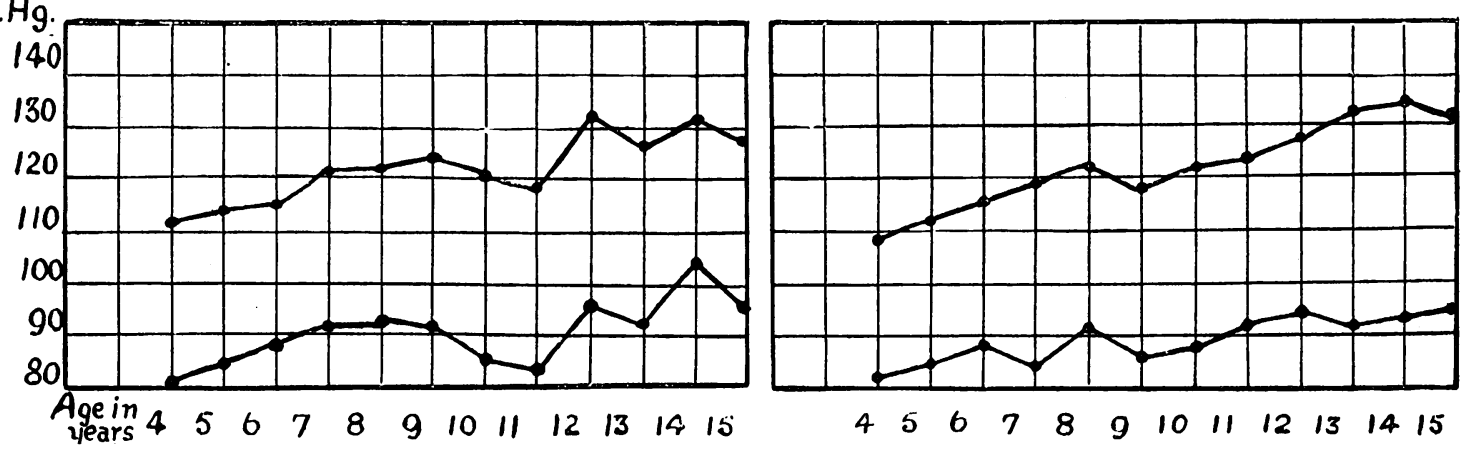

$\begin{array}{llllllllllll}4 & 5 & 6 & 7 & 8 & 9 & 10 & 11 & 12 & 13 & 14 & 15\end{array}$

Blood pressure measured with Vaquez-Laubry apparatus according to age. 
TABLE 2.

Arterinl tfension, according to ige, meastred by the P.achon apparatus.

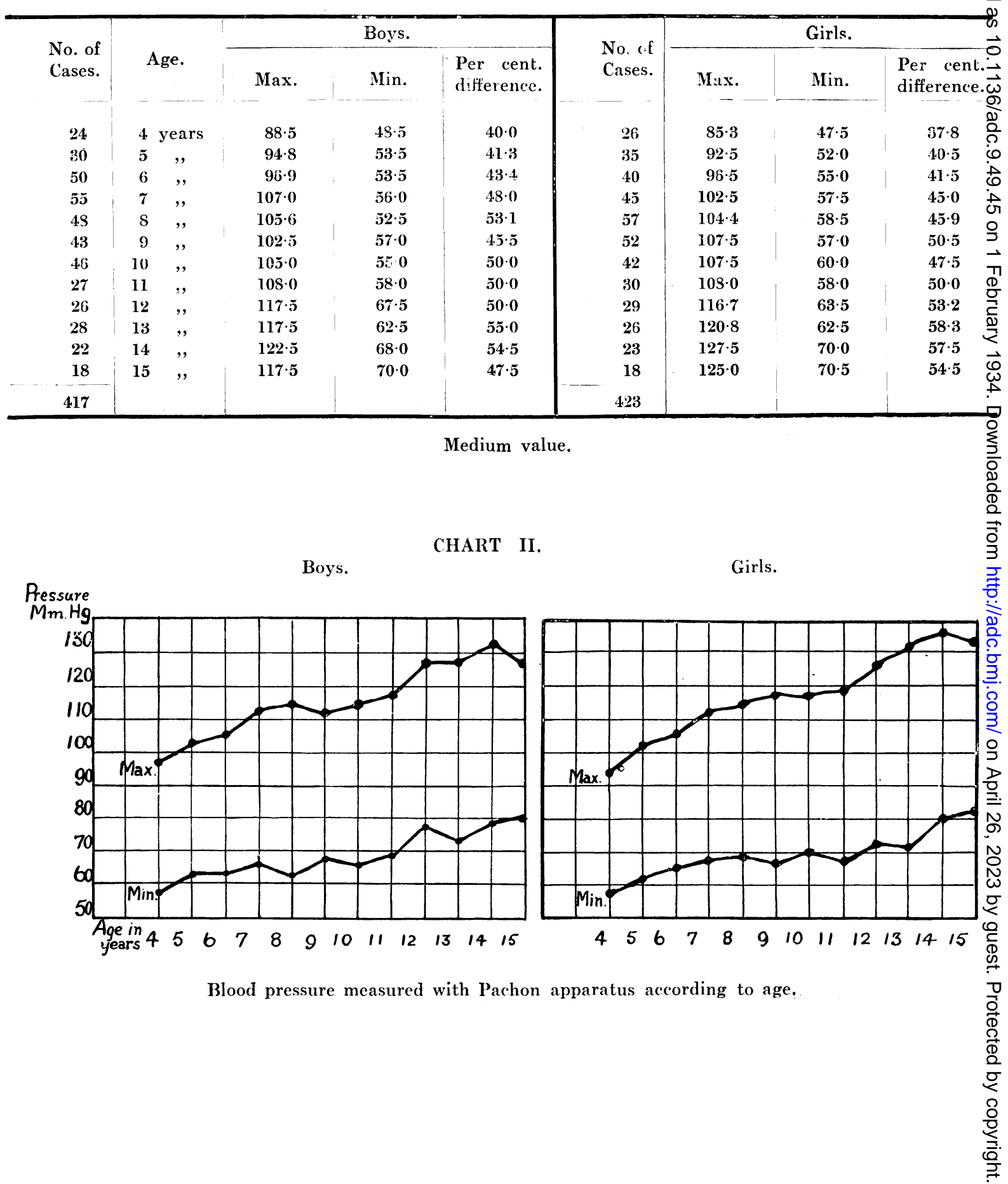


and 15 years is somewhat higher than in boys of the same age. It is also observed that the maximum pressure in boys at 12 and 14 years is the same, and that the minimum pressure in girls of 8 and 11 years is also the same.

In the course of these studies it was noticed that the use of the auscultatory method of estimation is not always satisfactory for taking the arterial tension in children. It is not easy to use, especially in measuring the minimum pressure in the very small child. An almost analogous remark has been made by Mouriquand and Barbier ${ }^{1}$, and Chabrun and Petrovitch ${ }^{2}$.

In comparing our results with those of other writers who have used the Vaquez-Laubry apparatus we find them nearly similar to those given by Chabrun and Petrovitch, and not very unlike those recorded by Melvin and Murray $^{3}$. In a series of 450 children Chabrun and Petrovitch found the maximum pressure in boys to vary between 100 and $120 \mathrm{~mm}$., and the minimum from 77.5 to $90 \mathrm{~mm}$.; in girls the maximum varied from 97.5 to $120 \mathrm{~mm}$., and the minimum from 77.5 to $90 \mathrm{~mm}$.

The Pachon oscillometer.-When measured with this apparatus the maximum pressure in boys is found to rise from $88.5 \mathrm{~mm}$. at 4 years to $122.5 \mathrm{~mm}$. at 14 years, and the minimum from 48.5 to $70 \mathrm{~mm}$. In girls the maximum pressure varies between $85 \mathrm{~mm}$. at 4 years and $125 \mathrm{~mm}$. at 14 years, and the minimum between $47.5 \mathrm{~mm}$. and $70.5 \mathrm{~mm}$. (Table 2 and Chart II).

\section{Discussion.}

On the basis of these facts it is seen that the maximum, minimum and differential arterial pressures in the Serbian child, as measured by Pachon's apparatus, increase with age in the case of both boys and girls; but it may be noted that the minimum pressure increases less rapidly than the maximum, and the maximum pressure increases nearly equally in both sexes. The maximum pressure is slightly higher in girls of 13, 14 and 15 years old. It is also interesting that the progression of the arterial tension is not absolutely regular during all the years of childhood. It rises slowly between the ages of 7 and 11 years, but between 11 and 14 years there is a more marked increase in the case of both girls and boys. It would also appear that the maximum, minimum and differential pressures in Serbian girls and boys are respectively equal at the age of 11 years.

It can also be stated that the maximum, minimum and differential pressures of normal Serbian children not only increase with age, but are likewise influenced by the height and weight of the child. Thus in the case of weak and thin children with retarded development the pressure increases more slowly than in children with a normal growth. These variations in the tension in relation to physical development are, however, not great and only affect the maximum pressure, the minimum pressure being practically equal irrespective of height and weight. 
The maximum pressure in girls of 13, 14 and 15 years is higher than in boys of corresponding ages. This is probably due to the fact that Serbian girls of this age are generally more developed than boys of the same age.

These results are analogous to those obtained by Chabrun and Petrovitch with the same apparatus. According to these writers the maximum pressure in boys between 4 and 15 years steadily rises from 87.5 to $120 \mathrm{~mm}$., and the minimum from 47.5 to $65 \mathrm{~mm}$.; in girls the maximum varies between 82.5 and $120 \mathrm{~mm}$. and the minimum between 43 and $70 \mathrm{~mm}$. Likewise our results are the same as those obtained by Garot and Schwers ${ }^{4}$. According to their observations in children between the ages of 2 and 12 years the maximum pressure varies from 90 to $110 \mathrm{~mm}$. and the minimum from 67.5 to $77.5 \mathrm{~mm}$. On the other hand, our results are lower than the high figures given by Koessler.

\section{Conclusions.}

1. The Pachon oscillometer is an excellent apparatus for taking the arterial pressure of children as it is easy to use and the results are very exact. The auscultatory method is somewhat more difficult in the case of children, especially when the minimum blood pressure in very young children is required.

2. The study of blood pressure in children requires much time and patience as it should be based on a great number of observations. The measurements should be taken under analogous technical conditions.

3. To obtain the approximate average value of the arterial tension of children, it is necessary to take into consideration the age and development of the child, the climatic conditions and the food, and also the technical difficulties.

4. The present observations are based on a sufficient number of cases and have been used to establish a chart of the normal arterial pressure in Serbian children between the ages of 4 and 15 years.

5. The curves attached to this work indicate the average value of the blood pressure in the normal Serbian child between the ages of 4 and 15 years. These curves are based on 840 observations and the measurements were taken by modern apparatus and under the same technical conditions. These curves have an interest from a physiological and a clinical point of view.

6. The arterial pressure in Serbian children of both sexes is not uniform but increases with the age, weight and height of the child. Between the ages of 7 and 11 years the blood pressure increases very slightly, but there is a decided increase in the pressure between 11 and 14 years, the age of puberty. 


\section{REFERENGES.}

1. Mouriquand, G., \& Barbier, J., Lyon méd., Lyon, 1922, CXXXI, 1073.

2. Chabrun, J., \& Petrovitch, Y., Arch. d. méd. d. enfants, Paris, 1930, XXXIII, 601.

3. Melvin, G. S., \& Murray, J. R., Brit. Med. J., Lond., 1915, i, 669.

4. Garot, L., \& Schwers, H., Rev. franc, de Pédiat., 1926, II, 36.

5. Koessler, L., Thése de Paris, 1912, No. 144. 